\title{
Laser-driven coating of vertically aligned carbon nanotubes with manganese oxide from metal organic precursors for energy storage
}

\author{
A. Pérez del Pino ${ }^{1, *}$, E. György ${ }^{1,2}$, I. Alshaikh ${ }^{3,4}$, F. Pantoja-Suárez ${ }^{3,4,5}$, J. L. Andújar ${ }^{3,4}$, \\ E. Pascual ${ }^{3,4}$, R. Amade ${ }^{3,4}$, E. Bertran-Serra ${ }^{3,4}$
}

${ }^{1}$ Instituto de Ciencia de Materiales de Barcelona, Consejo Superior de Investigaciones Científicas (ICMAB-CSIC), Campus UAB, 08193 Bellaterra, Spain

${ }^{2}$ National Institute for Lasers, Plasma and Radiation Physics, P. O. Box MG 36, 77125 Bucharest, Romania

${ }^{3}$ Departament de Física Aplicada, Universitat de Barcelona, C/Martí i Franquès 1, 08028 Barcelona, Spain

${ }^{4}$ Institute of Nanoscience and Nanotechnology (IN2UB), Universitat de Barcelona, Avda. Joan XXIII, s/n, 08028 Barcelona, Spain

${ }^{5}$ Departamento de Materiales, Facultad de Ingeniería Mecánica, Escuela Politécnica Nacional, Ladrón de Guevara, E11-253, Quito, Ecuador

*Corresponding author (Telephone:+34 935801853; E-mail: aperez@icmab.es)

\begin{abstract}
Carbon nanotubes-transition metal oxide systems are intensively studied due to their excellent properties for electrochemical applications. In this work, an innovative procedure is developed for the synthesis of vertically aligned multi-walled carbon


nanotubes (VACNTs) coated with transition metal oxide nanostructures. VACNTs are grown by plasma enhanced chemical vapor deposition and coated with a manganesebased metal organic precursor (MOP) film based on manganese acetate solution. Subsequent UV pulsed laser irradiation induces the effective heating-decomposition of the MOP leading to the crystallization of manganese oxide nanostructures on the VACNT surface. The study of the morphology, structure and composition of the synthesized materials shows the formation of randomly oriented $\mathrm{MnO}_{2}$ crystals, with few nanometers in size, and to their alignment in hundreds of nm long filament-like structures, parallel to the CNT's long axis. Electrochemical measurements reveal a significant increase of the specific capacitance of the $\mathrm{MnO}_{2}-\mathrm{VACNT}$ system (100 F g $\mathrm{g}^{-1}$ ) as compared to the initial VACNT one $\left(21 \mathrm{~F} \mathrm{~g}^{-1}\right)$.

Keywords: vertically aligned carbon nanotubes, $\mathrm{MnO}_{2}$, hybrid nanocomposite, laser irradiation, energy storage.

\section{Introduction}

Transition metal oxide (TMO) materials exhibit fascinating physical and chemical properties which make them essential in several applications [1]. Particularly, TMO nanostructures are investigated to be used in many electrochemical applications as sensors, electrocatalysts for oxygen reduction reactions, batteries and supercapacitors [2-5]. Manganese dioxide $\left(\mathrm{MnO}_{2}\right)$ is a highly stable and abundant oxide with excellent physico-chemical properties [6, 7]. In addition, due to its environmental friendliness and low cost, $\mathrm{MnO}_{2}$ is an excellent candidate for a wide range of electrochemical applications including catalysis, biosensing, and energy storage [8, 9]. In particular, 
$\mathrm{MnO}_{2}$ has been extensively studied as a high specific capacitance electrode material in high-performance supercapacitors due to its large operating voltage, appearing as a potential electrode material for a cheaper replacement of $\mathrm{RuO}_{2}$ in supercapacitors. However, $\mathrm{MnO}_{2}$ exhibits low conductivity which significantly hampers its electrochemical performance and application in bulk conformation.

On the other hand, carbon nanotubes (CNTs) and graphene materials can exhibit large electric conductivity and can sustain extremely high electric current densities (up to $4 \times 10^{9} \mathrm{~A} \mathrm{~cm}^{-2}$ in metallic CNTs). Moreover, they have other outstanding properties for electrochemical applications such as stability and huge active surface area (with enhancement factors of more than 60) [10], as well as high hardness and flexibility. Thus, the synthesis of graphene-like and carbon nanotubes coated with TMO materials, and in particular $\mathrm{MnO}_{2}$, is being thoroughly investigated for the development of highperformance devices in diverse applications such as supercapacitors [11-15], batteries $[16,17]$, microbial fuel cells $[18,19]$ or sensors [20]. It is worth noticing that layers composed of vertically aligned CNT (VACNT) can show larger active area and lower contact resistance with the substrate than those composed of randomly oriented CNTs. On the other hand, besides the low electric conductivity, $\mathrm{MnO}_{2}$ exhibits slow proton and cation diffusion rates, limiting the electrochemical reaction to its surface layer. Then, ultrathin oxide coatings of only a few nanometers of thickness are convenient to be synthesized on CNT surfaces for obtaining both high capacitance and high chargedischarge rates.

Laser radiation is characterized by its highly coherent, monochromatic and intense nature, and it can be delivered in very short pulses. Thus, materials submitted to intense laser pulses can suffer significant structural and compositional transformations often out of thermodynamic equilibrium [21-25]. These phase changes can be induced by either 
high-temperature thermal cycles or by direct photoreactions (or a combination of them). Recently, the growth of oxide thin films through the laser treatment of metal organic precursors (MOP) appeared as a versatile, fast, environmentally-friendly, cost-effective and easily-scalable method for synthesizing practical nanomaterials. Thus, direct laser irradiation of MOP coatings led to the development of diverse types of functional polycrystalline oxides [26-30]. Remarkably, the epitaxial growth of functional oxide films was also achieved [25, 31-36].

Herein, we report a novel approach for obtaining hybrid $\mathrm{MnO}_{2}$-coated VACNTs. The VACNTs, grown by plasma enhanced chemical vapor deposition (PECVD), are coated by Mn-based MOP and subsequently irradiated by nanosecond pulsed UV laser irradiation. The structural-compositional studies reveal that the UV radiation leads to the MOP decomposition and recrystallization to $\mathrm{MnO}_{2}$ nanostructures situated on the surface of the CNTs. Besides, the nanostructured oxide coating of the VACNTs leads to a significant improvement of the energy storage capability of the VACNT system. This method opens a window to the facile and cost-effective synthesis of nanocarbon-TMO hybrid materials. Conversely to the laser processing of VACNTs coated with TMO nanoparticles [37], the irradiation of MOP-VACNT systems can lead to enhanced and more uniform coating of dense VACNT forests.

\section{Experimental}

Material synthesis

Fig. 1 shows a scheme of the synthesis procedure. Firstly, VACNTs are grown on silicon substrate by plasma enhanced chemical vapor deposition (PECVD) in a multiple 
chamber reactor with sputtering heads, an annealing port and a cathode for PECVD processes. First, a $3 \mathrm{~nm}$ layer of Fe catalyst is sputtered by RF-plasma under Ar (128 sccm, $2 \mathrm{~Pa}$ ). Then, the sample is annealed up to $680^{\circ} \mathrm{C}$ with a ramp time of $750 \mathrm{~s}$ in a $\mathrm{H}_{2}$ atmosphere (100 sccm, $200 \mathrm{~Pa}$ ). Afterwards, the temperature is kept constant during 120 $\mathrm{s}$ to promote the formation of iron nanoislands, which act as seeds for the growth of CNTs. Subsequently, the pressure of the reactor is lowered to $100 \mathrm{~Pa}$ followed by the introduction of $\mathrm{NH}_{3}(100 \mathrm{sccm})$ and a RF plasma (50W) is ignited. Then, the carbon source $\left(\mathrm{C}_{2} \mathrm{H}_{2}, 50 \mathrm{sccm}\right)$ is introduced and the CNTs growth takes place during $900 \mathrm{~s}$. Finally, VACNTs are obtained with an areal density of about $140 \mu \mathrm{g} / \mathrm{cm}^{2}$. From the analysis of the SEM images, it is observed that the CNTs grow vertically aligned, with high density, a height of about 10-15 $\mu \mathrm{m}$ and a diameter between 20-40 nm (Fig. S1, Supplementary material).

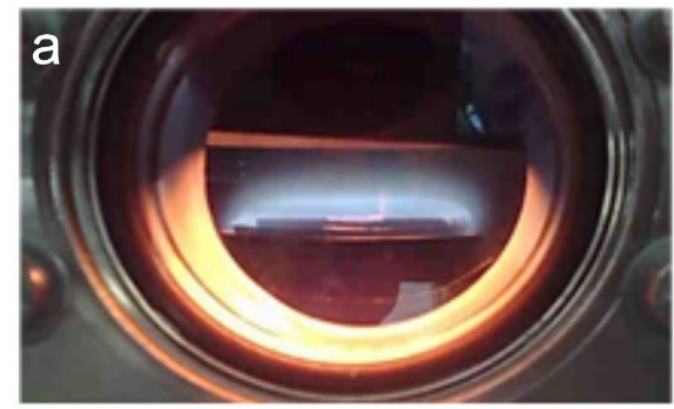

b

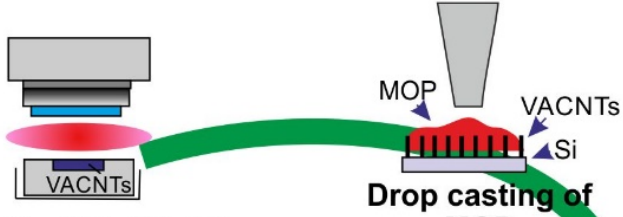

Growth of VACNT/Si by PECVD

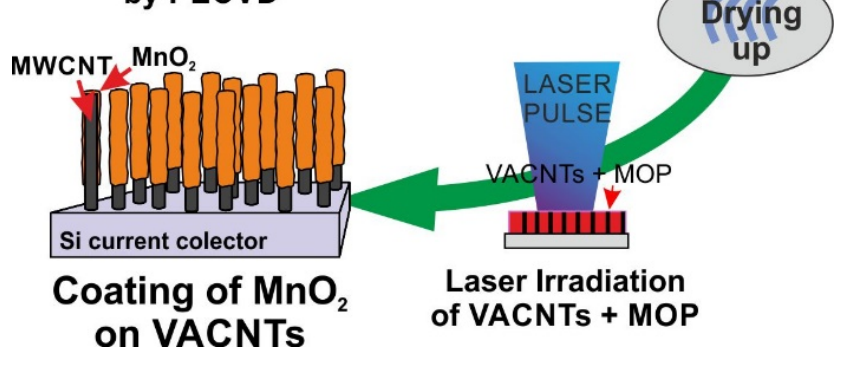


Fig 1. (a) View of the annealing port and the cathode for PECVD processing of VACNTs. (b) Sketch of the synthesis method for obtaining VACNT forests coated with nanostructured $\mathrm{MnO}_{2}$.

Metal organic precursor (MOP) solutions are obtained by dispersing manganese (II) acetate (Sigma-Aldrich) in distilled water at 0.02, 0.05 and 0.1 wt.\% concentration. After thorough stirring and sonication, a volume of $4 \mu \mathrm{L}$ per $\mathrm{mm}^{2}$ of sample area is casted on VACNTs surface. The samples are left at $50{ }^{\circ} \mathrm{C}$ temperature until the evaporation of the solvent is completed. Afterwards, the coated samples are submitted to pulsed laser irradiation (266 nm wavelength, 5 ns- pulse duration, $10 \mathrm{~Hz}$ repetition rate) through a Quantel Nd:YAG Brilliant B system. The laser beam spatial distribution is shaped to homogeneous squared one by using a Galilean telescope and a squared mask. The beam is focused onto the samples surface by a convergent lens to $1 \mathrm{~mm}^{2}-$ sized square spots. Larger areas are laser treated by irradiating adjacent locations with a separation distance of $1 \mathrm{~mm}$ between the centers of the spots. The treatments are performed in air at atmospheric pressure through the accumulation of 500-1000 laser pulses for each $1 \mathrm{~mm}^{2}$ square spot area at laser fluence values in the range of 40-200 $\mathrm{mJ}$ $\mathrm{cm}^{-2}$.

\section{Characterization}

The morphology of the obtained samples was characterized by field emission scanning electron microscopy (SEM) with a QUANTA 200 FEG-ESEM microscope and extreme high resolution SEM (XHR SEM) by using a Magellan 400L system, both from FEI. The structural study was performed by high resolution transmission electron 
microscopy (HRTEM) and high angle annular dark field scanning TEM (HAADFSTEM) using a Tecnai F20 equipment (FEI). The compositional study of the obtained materials was carried out by energy-dispersive X-ray spectroscopy (EDX) with the FEI Tecnai F20 microscope, and Raman spectroscopy by means of a LabRAM 800 system from Horiba Jobin Yvon. The measurements were performed in spots of ca. $500 \mathrm{~nm}$ in diameter with a $532 \mathrm{~nm}$ laser wavelength $(1.5 \mathrm{~mW})$. The spectra were acquired in the $200-2000 \mathrm{~cm}^{-1}$ range, and an average of 3 spectra of $20 \mathrm{~s}$ of acquisition time was carried out for improving the signal. Finally, the optical absorption of the precursor solutions was analyzed by UV-Visible-IR spectrophotometry by means of a double beam Varian Cary 500 system. Absorbance (A) spectra of the liquid solutions in quartz cuvettes were acquired, and the absorption coefficient $(\alpha)$ was calculated by using the formula:

$$
\alpha=\frac{1}{d} \operatorname{Ln}\left(10^{A}\right)
$$

Being $d$ the optical pathway through the liquid solution in the cuvette.

The electrochemical properties of the samples were analyzed by cyclic voltammetry (CV) and electrochemical impedance spectroscopy (EIS) using a potentiostat / galvanostat (AutoLab, PGSTAT30, USA). The samples were used as working electrode with a geometrical area of $0.57 \mathrm{~cm}^{2}$ in a three-electrode cell. The measurements were performed with a $\mathrm{Ag} / \mathrm{AgCl}$ (3M KCl internal solution) reference electrode and a Pt-ring counter electrode in a $1 \mathrm{M} \mathrm{Na}_{2} \mathrm{SO}_{4}$ aqueous solution at room temperature.

The average specific capacitance of the samples was calculated at different scan rates according to the following equation:

$$
C_{s}=\frac{q_{a}+\left|q_{c}\right|}{2 \Delta V m}
$$

where $C_{s}$ is the specific capacitance in $\mathrm{F} \mathrm{g}^{-1}, q_{a}$ and $q_{c}$ are the anodic and cathodic charge, respectively, in $\mathrm{C} . \Delta V$ is the voltage window in $\mathrm{V}$ and $m$ the mass of the active 
material in g. The electrochemical series resistance (ESR) of the samples was determined from the $\mathrm{x}$-axis interception of the EIS spectra in the Nyquist plot.

\section{Results and discussion}

SEM exploration was used to reveal the morphology of the VACNTs after the casting of the Mn acetate solution (Fig. 2). As observed in Fig. 2a, secondary electron images disclose that the carbon nanotubes, $10-15 \mu \mathrm{m}$ in length, are vertically aligned, straight and successfully coated with the solution up to about $5 \mu \mathrm{m}$ in depth. This fact is corroborated through backscattered electron images, where Mn material appears much brighter than carbon and its spatial distribution is easily discernible (Fig. 2b). VACNTs seem to be uniformly coated with the precursor in all cases. However, while individual CNTs are clearly discernible when covered with 0.02 wt.\% solution, exhibiting void spaces between CNTs, the samples coated with 0.05 and 0.1 wt.\% solutions show a complete filling of the spaces between CNTs, leading to the formation of a compact film (Fig. 2c, d). 

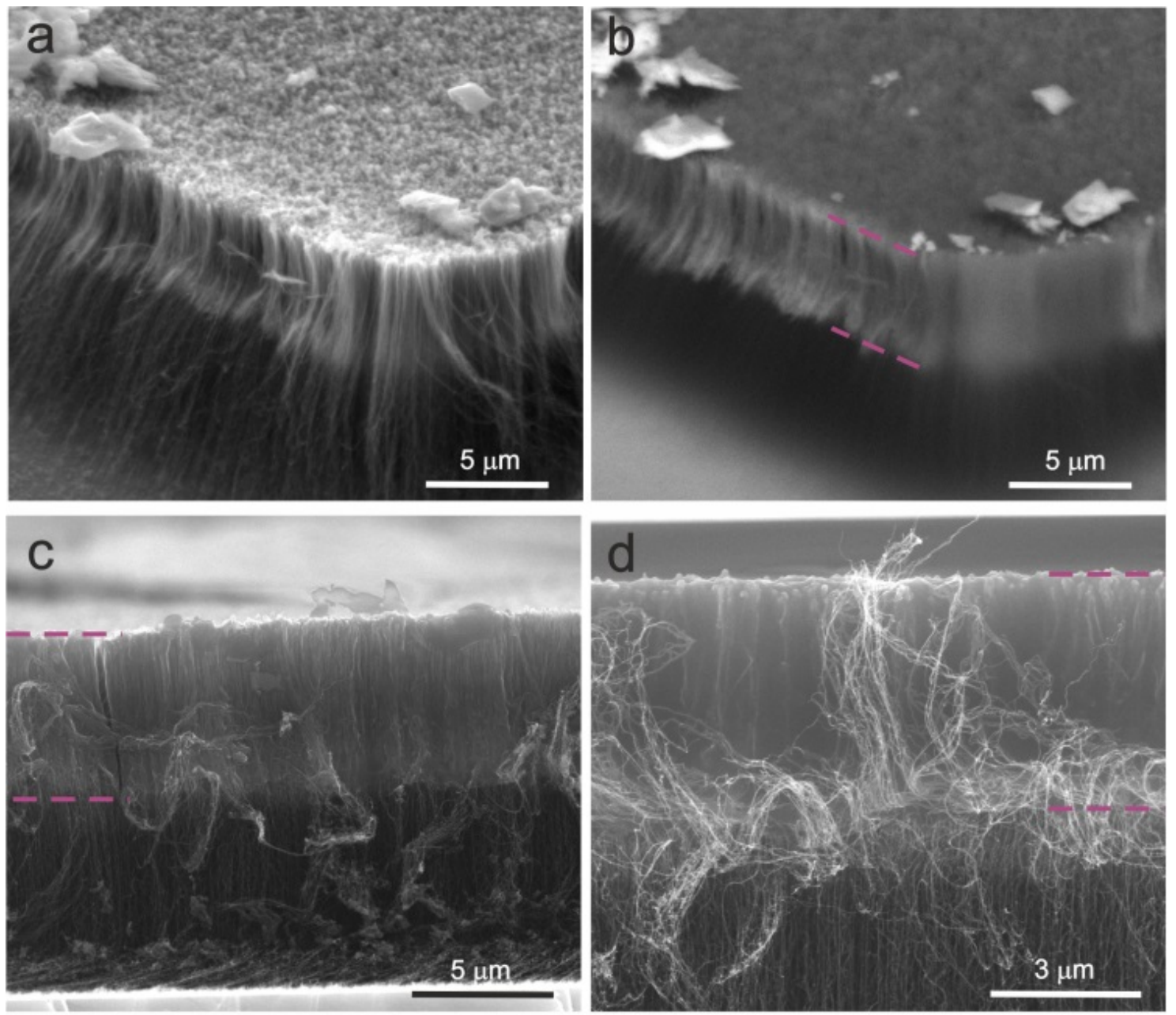

Fig 2. SEM pictures of VACNTs impregnated with manganese acetate solution. Images taken with (a) secondary and (b) backscattered electrons of sample obtained with 0.02 wt.\% precursor concentration, and secondary electron images taken at samples impregnated with (c) 0.05 and (d) 0.1 wt.\% concentration. The dashed lines indicate the coated depth.

As previously stated, only ultrathin layers of $\mathrm{MnO}_{2}$ are valuable for electrochemical applications, due to its low electronic and ionic conductivity. In addition, void spaces between CNTs are required to allow the electrolyte reaching the largest possible surface of CNTs. Furthermore, electrolyte ions should be able to easily diffuse in and out of the electrode material through the void channels for improving redox reactions rates. Then, 
further studies are focused in CNTs coated with 0.02 wt.\% manganese acetate solution which show thin enough coating on the VACNT surface. High resolution SEM inspection of the samples' top surface shows that the VACNTs, about 20-50 nm in diameter, are separated around 20 to $200 \mathrm{~nm}$ (Fig. 3a). Moreover, VACNTs show a droplet-like cap on top of them reaching about $100 \mathrm{~nm}$ in size. The droplet-like caps, not observed on CNTs prior to solution deposition, might be composed by manganese precursor. Laser processing of the coated VACNTs leads to different morphological evolution depending on the incident laser fluence and the accumulated number of pulses. Laser irradiation with fluences below ca. $100 \mathrm{~mJ} \mathrm{~cm}^{-2}$ does not induce significant morphological changes (Fig. 3b). Nevertheless, the accumulation of more than 500 laser pulses at $100 \mathrm{~mJ} \mathrm{~cm}^{-2}$ mainly reveal thinner VACNTs without capping material, though some capped CNTs can be still identified (Fig. 3c, d). The number of uncapped CNTs increases with the number of accumulated pulses. Finally, laser irradiation with fluences higher than $120 \mathrm{~mJ} \mathrm{~cm}^{-2}$ leads to the partial meltingcoalescence of the CNTs, and further ablation with the accumulation of pulses (Fig. S2, Supplementary material). Therefore, the range of laser parameters in order to induce a morphologic change of the coated CNTs but not their damaging by meltingvaporization processes appears to be rather narrow. 

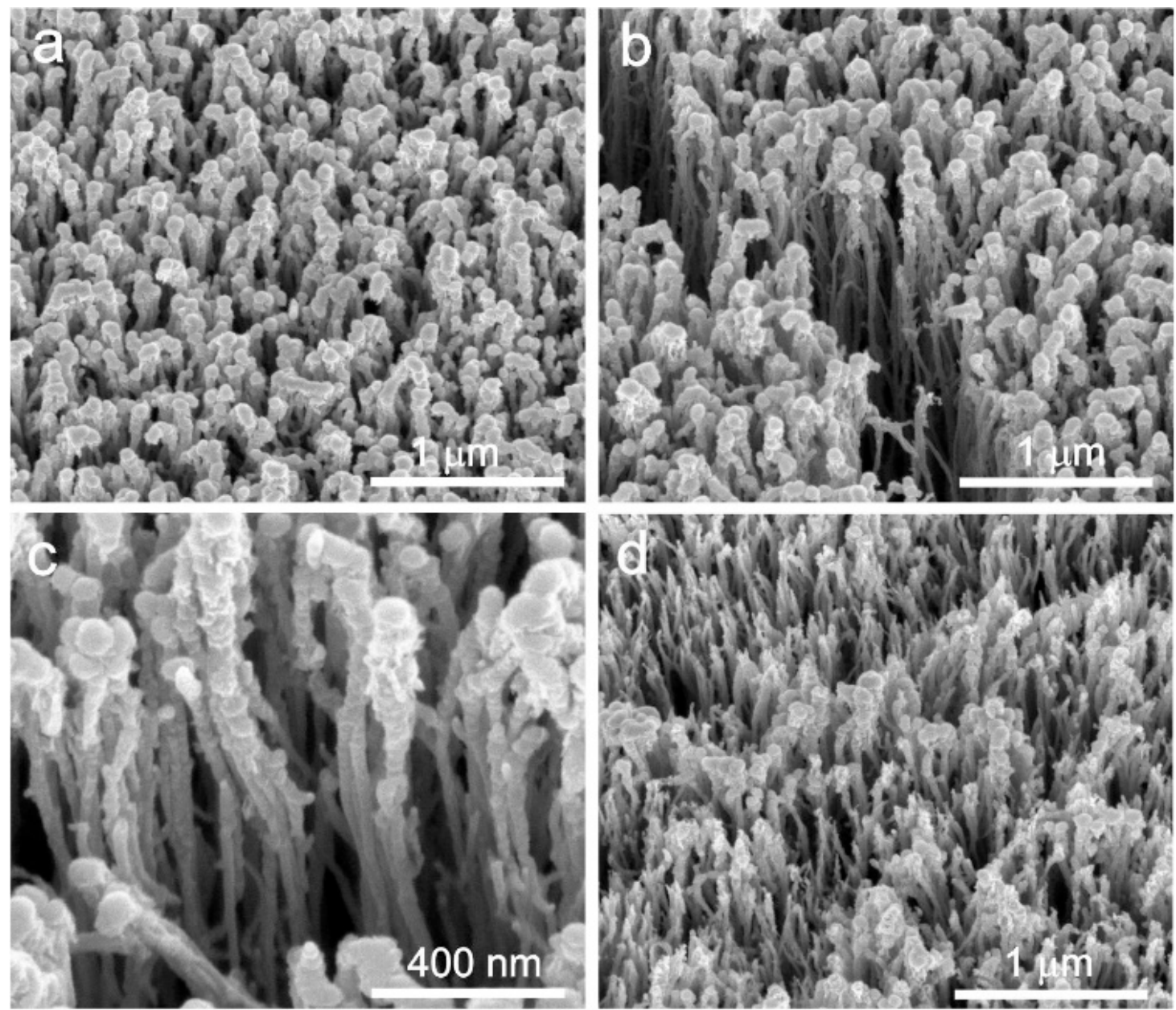

Fig 3. SEM pictures of VACNTs impregnated with 0.02 wt.\% manganese acetate solution (a) as deposited, and irradiated with (b) $50 \mathrm{~mJ} \mathrm{~cm}{ }^{-2}, 1000$ pulses, (c) $100 \mathrm{~mJ}$ $\mathrm{cm}^{-2}, 500$ pulses, and (d) $100 \mathrm{~mJ} \mathrm{~cm}^{-2}, 1000$ pulses.

A detailed investigation of the structure of the irradiated MOP-VACNTs is carried out by HAADF-STEM in Z-contrast mode. Thus, the intensity contrast in the images is related to the atomic number. Fig. 4 shows STEM images of nanotubes irradiated by accumulation of 1000 pulses with $80 \mathrm{~mJ} \mathrm{~cm}^{-2}$ laser fluence. These images reveal straight beams of carbon nanotubes, about $50 \mathrm{~nm}$ in diameter, which present bright nanostructures corresponding to Mn-rich material on their surface (Fig. 4a). Interestingly, higher resolution studies show two types of Mn nanostructures: round 
crystallites about few $\mathrm{nm}$ in size, and parallel hundreds of $\mathrm{nm}$ in length filament-like structures aligned to the CNT's long axis (Fig. 4b). These structures have a diameter of about $5 \mathrm{~nm}$. The round nanostructures appear randomly distributed on the long ones. EDX spectra acquired in hundreds of nanometers square areas reveal mainly the presence of carbon from the CNTs, manganese and oxygen, indicating the adhesion of manganese oxide to the surface of the CNTs (Fig. 5a). Copper and silicon signal is originated from the TEM samples' holder. Fig. 4c shows a STEM image of capping material located at the top of the VACNTs. In these regions, randomly distributed round nanostructures are mainly observed, revealing that manganese-rich nanostructures are also present even in the cap material of the VACNTs. High resolution TEM characterization is also carried out in order to determine the crystalline nature of the manganese oxide coating. As shown in Fig. 5b, crystals of few to tens of nanometers in size appear attached to the VACNT's surface. The graphitic planes of the CNTs appear clearly defined along the nanotube, showing some crystalline defects especially at the cap of the CNTs (Fig. S3, Supplementary material). Selective area electron diffraction (SAED) images obtained in hundreds to thousands of $\mathrm{nm}^{2}$ areas reveal the presence of relatively thick circular patterns as well as well-defined spots. The circular patterns correspond to interplanar distances of (i) $3.4 \AA$, (ii) $2.1 \AA$, (iii) $1.7 \AA$ and (iv) $1.2 \AA$, being the uncertainty about $0.1 \AA$. Thus, (i) would correspond to the spacing between graphitic shells in the CNTs. The intensity asymmetry observed in this ring accounts for the parallel alignment of the VACNT beams. The rest of the rings can be assigned to different orientations of $\alpha-\mathrm{MnO}_{2}$ structure (JCPDS-00-044-0141): (ii) (301), (iii) contribution of (600), (440) and (411) planes, and (iv) (332). Alternatively, these rings could be assigned to (ii) (300), (iii) (160), and (iv) (450) reflections of $\gamma-\mathrm{MnO}_{2}$ (JCPDS00-014-0644). The thick circular rings denote a randomly oriented configuration of 
$\mathrm{MnO}_{2}$ nanocrystals with different degree of crystallinity. However, the bright spots, almost all of them appearing randomly distributed on the $2.1 \AA$ ring, reveal the formation of highly crystalline oriented nanostructures. The ring (iii), corresponding to $1.7 \AA$ spacing, presents an intensity asymmetry in the same direction of that showed by (i), which corresponds to the VACNTs. Therefore, it could be pointed out that the ring (iii) is originated by the observed long $\mathrm{MnO}_{2}$ nanostructures parallel to the long axis of the VACNTs (Fig. 4b). No additional intensity asymmetries are observed in the rest of diffraction rings. This result suggests that the aligned $\mathrm{MnO}_{2}$ nanostructures would have a preferential orientation. 


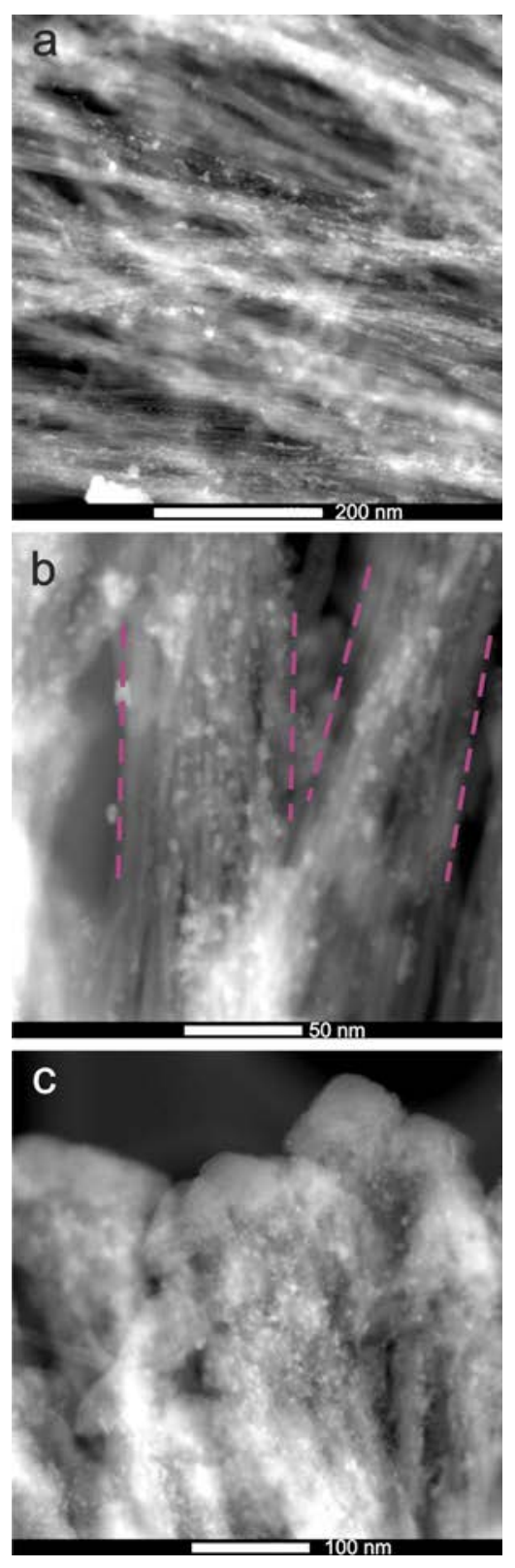

Fig 4. HAADF-STEM images of VACNTs coated with 0.02 wt.\% manganese acetate solution and laser irradiated with $80 \mathrm{~mJ} \mathrm{~cm}^{-2}$ and 1000 pulses. (a) and (b) show the body and (c) the cap material at the top of the VACNTs. The dashed lines in (b) define the boundaries of two carbon nanotubes.

Raman spectroscopy analyses are likely to provide further information about the structure of the $\mathrm{MnO}_{2}$-coated VACNTs. Fig. 6 displays representative spectra of non- 
irradiated MOP-VACNT sample and one irradiated by accumulation of 1000 pulses with $100 \mathrm{~mJ} \mathrm{~cm}{ }^{-2}$ laser fluence. The spectra show a typical configuration of bands from MWCNTs. These bands, centered at ca. 1100, 1350 and $1580 \mathrm{~cm}^{-1}$, respectively correspond to I, D and G signals [38]. The I band, which has been previously assigned to diamond-like $\mathrm{sp}^{3}$ and $\mathrm{sp}^{2}$ carbon bonds in trans-polyacetylene confined in nanodiamond [39], can be associated to the presence of disordered graphitic carbons in CNT structure. D band is assigned to A1g symmetry breathing mode activated by defects-disorder at aromatic rings, whereas $G$ band is generated by $\mathrm{sp}^{2}$-hybridized carbon atoms. The D/G intensity ratio is frequently used as a figure of merit for the defect content of the CNTs. Accordingly, non-irradiated MOP-VACNT sample exhibits a D/G ratio of ca. 0.97. Irradiation of the samples with $50 \mathrm{~mJ} \mathrm{~cm}^{-2}$ and 1000 pulses does not lead to a significant change of $\mathrm{D} / \mathrm{G}$ ratio (ca. 0.99). With the increase of the laser fluence, the D/G ratio slightly increases (i. e. 1.05 with $100 \mathrm{~mJ} \mathrm{~cm}^{-2}, 500$ pulses) accounting for the laser-induced creation of defects (as observed by TEM). The gap between $\mathrm{D}$ and $\mathrm{G}$ peaks, $237 \pm 7 \mathrm{~cm}^{-1}$ in as grown VACNTs, do not significantly vary after the laser irradiations (all the values remain inside the initial experimental error). This confirms that the laser-induced disorder in the CNTs is relatively low. Finally, no bands from manganese oxides are detected, which should appear in the $500-650 \mathrm{~cm}^{-1}$ range [40]. This fact is probably due to the small amount and tiny size of the $\mathrm{MnO}_{2}$ crystallites. 

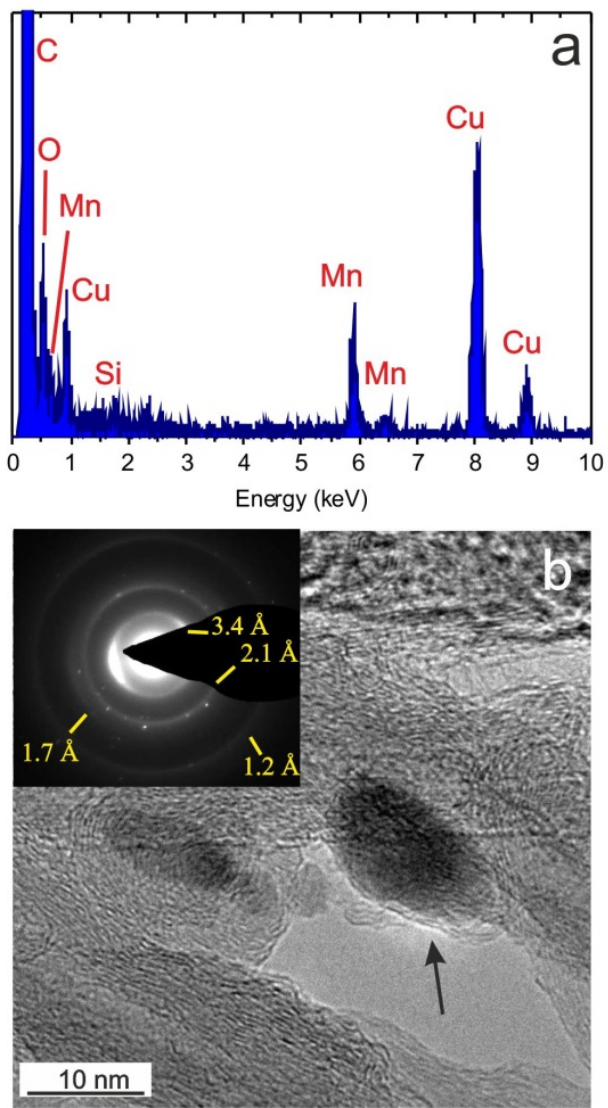

Fig 5. (a) EDX spectrum of the zone depicted in Fig 4b. (b) HRTEM image and diffraction pattern in a sample irradiated with $80 \mathrm{~mJ} \mathrm{~cm}^{-2}$ and 1000 pulses. The arrow points to a $\mathrm{MnO}_{2}$ nanostructure on a CNT. The diffraction pattern is acquired in a region of ca. $200 \times 200 \mathrm{~nm}^{2}$.

The absorption coefficient of Manganese (II) acetate precursor solution with $0.02 \mathrm{wt} . \%$ concentration versus wavelength, calculated from spectrophotometric measurements is shown in Fig. S4 (Supplementary material). As observed, the optical absorption of the MOP at the laser wavelength (266 nm) is negligible, being significant at wavelengths lower than $220 \mathrm{~nm}$. Therefore, it can be pointed out that the laser radiation is mainly absorbed by the VACNTs. The UV radiation excites the bonded electrons that constitute the CNT structure, probably accompanied by $\pi$ plasmons [41], leading to a substantial heating of the VACNTs. Accordingly, the higher the laser fluence, the higher the 
temperatures reached in the MOP-VACNT system. Previous works have demonstrated that UV laser pulses with fluences comparable to those used in this work can heat MWCNTs up to thousands of degrees during nanosecond times [38]. Therefore, the elevated temperatures would provoke the fast decomposition of the organic part of the MOP that coats the VACNTs, leading to the formation of amorphous-like or highly defective nanopolycrystalline manganese oxide. Since recrystallization of defective nanocrystallites is mainly governed by diffusion mechanisms, the temperature of the process is likely to play a key role. Therefore, beyond a threshold laser fluence, the recrystallization to randomly oriented $\alpha, \gamma-\mathrm{MnO}_{2}$ nanostructures takes place [42]. The varied degree of crystallinity of the nanocrystals would be related to the fast nature of the laser pulses and subsequent short thermal-induced cycles in the material [25]. However, the accumulation of laser pulses, which increases the "effective annealing time”, leads to further development of diffusion processes and subsequent improvement of the crystalline quality. On the other hand, previous works point out to the formation of large amount of crystalline defects and even the amorphization of CNTs submitted to UV laser irradiation [38, 43, 44]. Moreover, reported laser irradiation of CNTs exposed to air reveals oxidation processes which transform their structure to cauliflower-like one [45]. However, TEM and Raman studies revealed that under our irradiation conditions the crystallization of $\mathrm{MnO}_{2}$ on the VACNTs can take place without significant defect formation, being the main structure of the VACNTs preserved. 


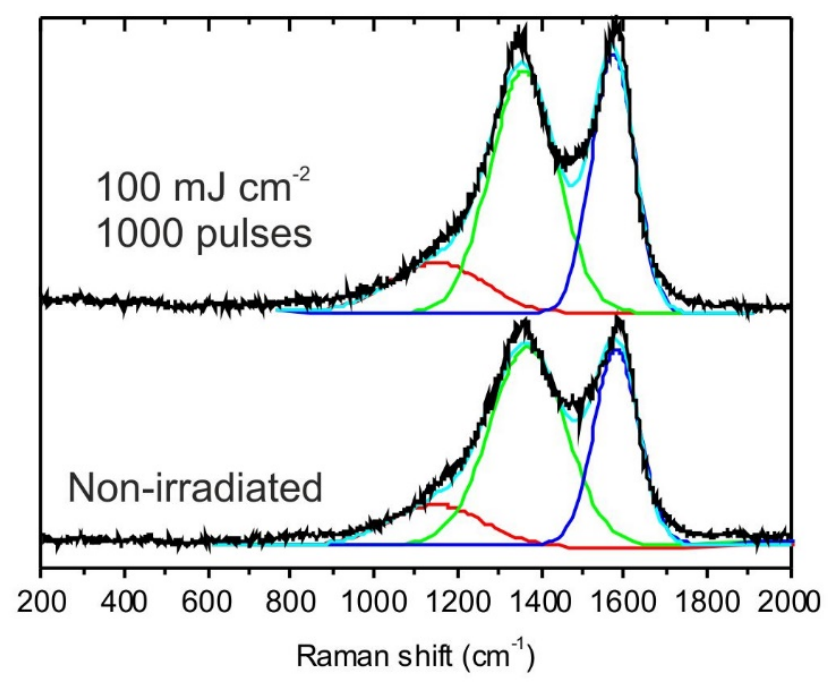

Fig 6. Raman spectra of VACNTs non-irradiated as well as laser irradiated by accumulation of 1000 pulses with100 $\mathrm{mJ} \mathrm{cm}^{-2}$.

The electrochemical behavior of VACNTs and $\mathrm{MnO}_{2}$ deposited on VACNTs is shown in Fig. 7. Cyclic voltammograms show a rectangular shape without clear redox peaks in the range of voltages analyzed (from 0 to $0.8 \mathrm{~V}$ vs. $\mathrm{Ag} / \mathrm{AgCl}$ ) which confirms the capacitive behavior of the samples (inset of Fig. 7a). As expected, the specific capacitance obtained from $\mathrm{CV}$ measurements is higher after deposition of $\mathrm{MnO}_{2}$ due to an increase in the pseudocapacitance of the electrode. Noticeably, at a scan rate of 10 $\mathrm{mV} \mathrm{s}^{-1}$, the specific capacitance of $\mathrm{MnO}_{2}-\mathrm{VACNTs}$ is about $100 \mathrm{~F} \mathrm{~g}^{-1}$, which is much higher than that obtained with bare VACNTs (21 $\mathrm{F} \mathrm{g}^{-1}$ ). The obtained specific capacitance values of $\mathrm{MnO}_{2}$-VACNTs are comparable to those of similar hybrid systems fabricated by conventional techniques as electrodeposition and chemical treatments $[14,15,46,47]$. Nonetheless, it has to be noted that the laser treatment is performed in a versatile way, at room temperature and without the use of toxic chemical products. In addition, from the Nyquist plot of the samples (Fig. 7b), the ESR of the electrodes can be determined, which is about 15 and $18 \Omega$ for $\mathrm{MnO}_{2}$-VACNTs and 
VACNTs, respectively. Thus, the speed of the charging process is slightly improved after the crystallization of $\mathrm{MnO}_{2}$. The ESR is a combination of the ionic resistance of the electrolyte, the intrinsic resistance of the substrate (current collector), VACNTs and the $\mathrm{MnO}_{2}$ materials, besides the contact resistances at the $\mathrm{MnO}_{2}$ / VACNTs / substrate interfaces. Additionally, the polarization resistance associated to the charge transfer reactions in the electrolyte $-\mathrm{MnO}_{2} /$ VACNT interfaces is lower in the $\mathrm{MnO}_{2}$-VACNTs system $(1 \Omega)$ than in the initial VACNT electrode (2.5 $\Omega$ ). This result accounts for the significant enhancement of the electron transfer kinetics between the electrolyte and the active material with the presence of the $\mathrm{MnO}_{2}$ nanostructures.
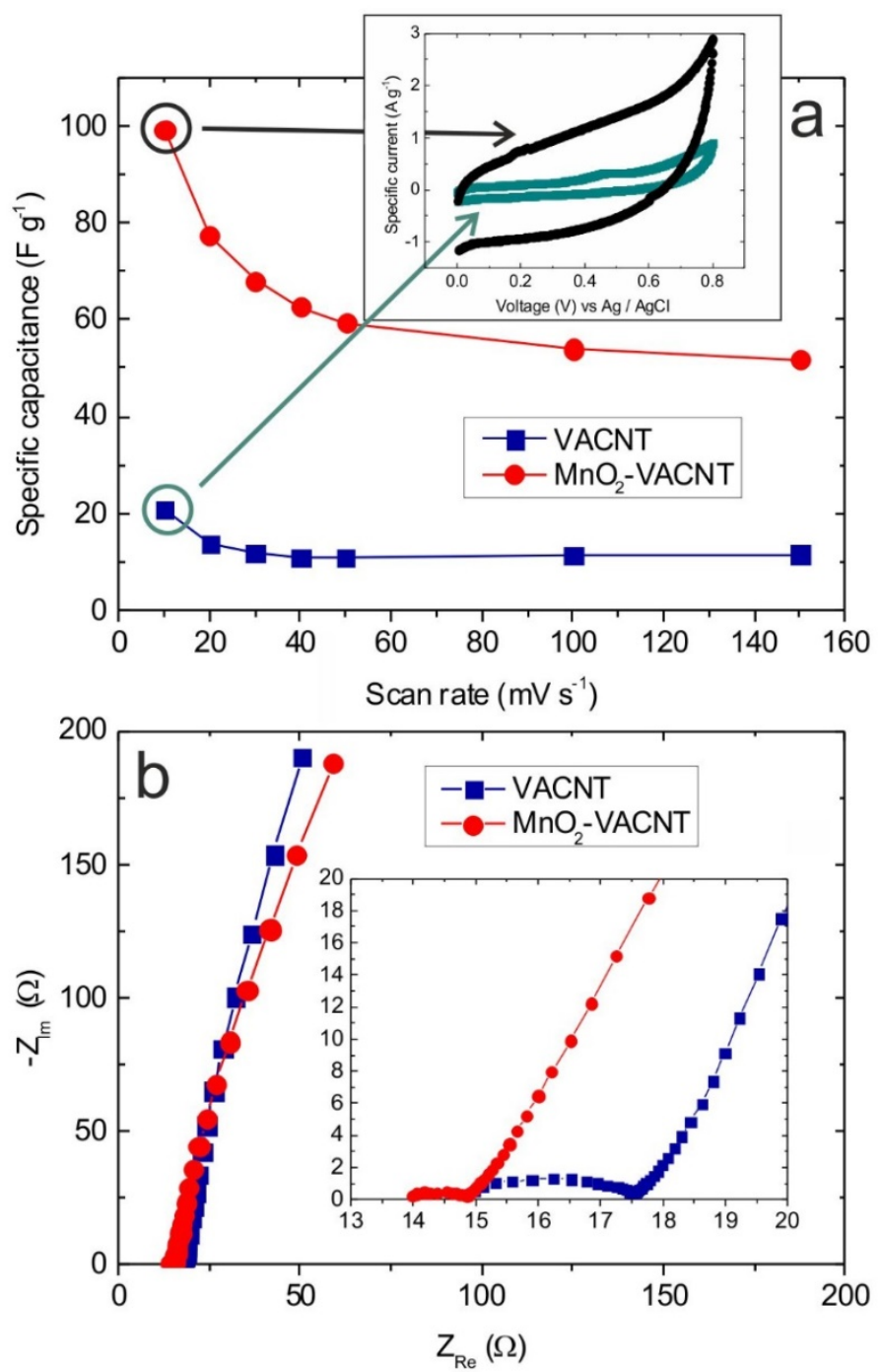
Fig 7. (a) Specific capacitance versus scan rate and (b) Nyquist plot of as grown VACNTs and $\mathrm{MnO}_{2}-\mathrm{VACNT}$ sample obtained by irradiation with $80 \mathrm{~mJ} \mathrm{~cm}^{-2}$ and 1000 pulses. Insets: (a) Cyclic voltammetries obtained at $10 \mathrm{mV} \mathrm{s}^{-1}$; (b) zoom at high frequency measurements.

\section{Conclusion}

In this work we report a versatile method for the fabrication of VACNT forests covered with transition metal oxide nanostructures. VACNTs coated with manganese (II) acetate precursor solution is irradiated with nanosecond pulsed UV laser radiation in order to induce the decomposition of the precursor and further crystallization to $\alpha, \gamma-\mathrm{MnO}_{2}$ nanostructures on the VACNTs surface. The oxides develop two types of configurations: hundreds of nanometers long structures oriented parallel to the long axis of the CNTs, and randomly distributed nanocrystals with up to $10 \mathrm{~nm}$ in size. Electrochemical characterization reveals better performance of the $\mathrm{MnO}_{2}-\mathrm{VACNT}$ system than the as grown (bare) VACNT one. This technique opens the door to the innovative development and large scale synthesis of a wide range of functional devices based on VACNTs coated with optically active, electrochemical, magnetic, ferroelectric or multiferroic coatings from metal organic precursors.

\section{Acknowledgements}

The authors acknowledge the financial support of the Spanish Ministry of Economy, Industry and Competitiveness under the projects MAT2010-20468, ENE2014-56109C3-1-R and ENE2014-56109-C3-3-R, as well as AGAUR (Generalitat de Catalunya) 
under the project 2014SGR984. ICMAB acknowledges financial support from the Spanish Ministry of Economy, Industry and Competitiveness, through the "Severo Ochoa” Programme for Centres of Excellence in R\&D (SEV- 2015-0496). I.A. and F.P. acknowledge finantial support of their PhD studies to MICINN and SENESCYT of the Ecuadorian Government, which provided financial support through its scholarship program for 2014, respectively. The authors would also like to thank the CCiT-UB for help with the structural and morphological characterization.

\section{Author's contributions}

Conceived the plan: AP, EG, JLA, EP, RA, EB-S; Performed the experiments: AP, EG, IA, FP-S, JLA, EP, RA, EB-S; Data analysis: AP, EG; Wrote the paper: AP. Authors have no competing financial interests.

\section{References}

1. Guo, T., et al., A comprehensive review on synthesis methods for transition-metal oxide nanostructures. Crystengcomm, 2015. 17(19): p. 3551-3585.

2. Yang, Z., et al., Facile Construction of Manganese Oxide Doped Carbon Nanotube Catalysts with High Activity for Oxygen Reduction Reaction and Investigations into the Origin of their Activity Enhancement. Acs Applied Materials \& Interfaces, 2011. 3(7): p. 2601-2606.

3. Reddy, A.L.M., et al., Coaxial MnO2/Carbon Nanotube Array Electrodes for HighPerformance Lithium Batteries. Nano Letters, 2009. 9(3): p. 1002-1006.

4. Liu, J.W., J. Essner, and J. Li, Hybrid Supercapacitor Based on Coaxially Coated Manganese Oxide on Vertically Aligned Carbon Nanofiber Arrays. Chemistry of Materials, 2010. 22(17): p. 5022-5030.

5. Nicole, C.S.P.B.M.P.L., Applications of advanced hybrid organic-inorganic nanomaterials: from laboratory to market. Chemistry Society Reviews, 2011. 40: p. 696753.

6. Zordan, T.A. and L.G. Hepler, Thermochemistry and Oxidation Potentials of Manganese and Its Compounds. Chemical Reviews, 1968. 68(6): p. 737-\&.

7. Subramanian, V., H.W. Zhu, and B.Q. Wei, Alcohol-assisted room temperature synthesis of different nanostructured manganese oxides and their pseudocapacitance properties in neutral electrolyte. Chemical Physics Letters, 2008. 453(4-6): p. 242-249.

8. $\mathrm{Xu}, \mathrm{M}$. , et al., Hydrothermal synthesis and pseudocapacitance properties of alphaMnO2 hollow spheres and hollow urchins. Journal of Physical Chemistry C, 2007. 111(51): p. 19141-19147. 
9. Devaraj, S. and N. Munichandraiah, Effect of crystallographic structure of MnO2 on its electrochemical capacitance properties. Journal of Physical Chemistry C, 2008. 112(11): p. 4406-4417.

10. Yarlagadda, V., et al., High Active Surface Area and Durable Multi-Wall Carbon Nanotube-Based Electrodes for the Bromine Reactions in H-2-Br-2 Fuel Cells. Journal of the Electrochemical Society, 2016. 163(1): p. A5134-A5143.

11. Chen, S., et al., Graphene Oxide-MnO2 Nanocomposites for Supercapacitors. Acs Nano, 2010. 4(5): p. 2822-2830.

12. He, Y.M., et al., Freestanding Three-Dimensional Graphene/MnO2 Composite Networks As Ultra light and Flexible Supercapacitor Electrodes. Acs Nano, 2013. 7(1): p. 174-182.

13. Wu, M.S., C.J. Lin, and C.L. Ho, Multilayered architecture of graphene nanosheets and MnO2 nanowires as an electrode material for high-performance supercapacitors. Electrochimica Acta, 2012. 81: p. 44-48.

14. Gu, T.L. and B.Q. Wei, Fast and stable redox reactions of MnO2/CNT hybrid electrodes for dynamically stretchable pseudocapacitors. Nanoscale, 2015. 7(27): p. 11626-11632.

15. Klankowski, S.A., et al., Higher-power supercapacitor electrodes based on mesoporous manganese oxide coating on vertically aligned carbon nanofibers. Nanoscale, 2015. 7(18): p. 8485-8494.

16. Salehi, M. and Z. Shariatinia, An optimization of MnO2 amount in CNT-MnO2 nanocomposite as a high rate cathode catalyst for the rechargeable Li-O-2 batteries. Electrochimica Acta, 2016. 188: p. 428-440.

17. Xu, D.W., et al., Preparation and Characterization of MnO2/acid-treated CNT Nanocomposites for Energy Storage with Zinc Ions. Electrochimica Acta, 2014. 133: p. 254-261.

18. Amade, R., et al., Vertically aligned carbon nanotubes coated with manganese dioxide as cathode material for microbial fuel cells. Journal of Materials Science, 2015. 50(3): p. 1214-1220.

19. Kalathil, S., et al., Enhanced Performance of a Microbial Fuel Cell Using CNT/MnO2 Nanocomposite as a Bioanode Material. Journal of Nanoscience and Nanotechnology, 2013. 13(11): p. 7712-7716.

20. Guo, C.Y., et al., 3D porous CNT/MnO2 composite electrode for high-performance enzymeless glucose detection and supercapacitor application. Sensors and Actuators B-Chemical, 2015. 206: p. 407-414.

21. Bäuerle, D., Laser Processing and Chemistry 2000, Berlin: Springer.

22. Maeng, J., et al., The effect of excimer laser annealing on ZnO nanowires and their field effect transistors. Nanotechnology, 2009. 20(9).

23. Perez del Pino, A., et al., Effects of pulsed laser radiation on epitaxial self-assembled Ge quantum dots grown on Si substrates. Nanotechnology, 2011. 22(29).

24. Aminuzzaman, M., A. Watanabe, and T. Miyashita, Direct writing of conductive silver micropatterns on flexible polyimide film by laser-induced pyrolysis of silver nanoparticledispersed film. Journal of Nanoparticle Research, 2010. 12(3): p. 931-938.

25. Queralto, A., et al., Ultrafast Crystallization of Ce0.9Zro.102-y Epitaxial Films on Flexible Technical Substrates by Pulsed Laser Irradiation of Chemical Solution Derived Precursor Layers. Crystal Growth \& Design, 2015. 15(4): p. 1957-1967.

26. Ghaith, E.S., et al., Apatite formation on rutile type TiO2 films formed by laser irradiation. Journal of Materials Science, 2006. 41(8): p. 2521-2524.

27. Baldus, O. and R. Waser, Laser crystallization studies of barium strontium titanate thin films. Journal of the European Ceramic Society, 2004. 24(10-11): p. 3013-3020.

28. Bharadwaja, S.S.N., et al., Excimer laser crystallized $(\mathrm{Pb}, \mathrm{La})(\mathrm{Zr}, \mathrm{Ti}) \mathrm{O}-3$ thin films. Journal of the American Ceramic Society, 2008. 91(5): p. 1580-1585.

29. Queralto, A., et al., Ultraviolet pulsed laser crystallization of Ba0.8Sr0.2TiO3 films on LaNiO3-coated silicon substrates. Ceramics International, 2016. 42(3): p. 4039-4047.

30. Tao, X.Y., et al., CO2 laser-induced crystallization of sol-gel-derived indium tin oxide films. Applied Physics a-Materials Science \& Processing, 2009. 96(3): p. 741-749.

31. Nishikawa, M., et al., Photoassisted chemical solution deposition method for fabricating uniformly epitaxial VO2 films. Applied Physics a-Materials Science \& Processing, 2010. 100(1): p. 297-303. 
32. Tsuchiya, T., T. Nakajima, and T. Kumagai, Influence of the laser wavelength on the epitaxial growth and electrical properties of La0.8SrO.2MnO3 films grown by excimer laser-assisted MOD. Applied Surface Science, 2009. 255(24): p. 9804-9807.

33. Nakajima, T., et al., Epitaxial Growth Mechanism for Perovskite Oxide Thin Films under Pulsed Laser Irradiation in Chemical Solution Deposition Process. Chemistry of Materials, 2008. 20(23): p. 7344-7351.

34. Nakajima, T., K. Shinoda, and T. Tsuchiya, UV-assisted nucleation and growth of oxide films from chemical solutions. Chemical Society Reviews, 2014. 43(7): p. 2027-2041.

35. Queralto, A., et al., Growth of ferroelectric BaO.8Sr0.2TiO3 epitaxial films by ultraviolet pulsed laser irradiation of chemical solution derived precursor layers. Applied Physics Letters, 2015. 106(26).

36. Queralto, A., et al., Ultrafast Epitaxial Growth Kinetics in Functional Oxide Thin Films Grown by Pulsed Laser Annealing of Chemical Solutions. Chemistry of Materials, 2016. 28(17): p. 6136-6145.

37. Perez del Pino, A., et al., Laser-induced nanostructuration of vertically aligned carbon nanotubes coated with nickel oxide nanoparticles. Journal of Materials Science, 2017. 52(7): p. 4002-4015.

38. Perez del Pino, A., et al., Ultraviolet pulsed laser irradiation of multi-walled carbon nanotubes in nitrogen atmosphere. Journal of Applied Physics, 2014. 115(9).

39. Ferrari, A.C. and J. Robertson, Origin of the 1150-cm(-1) Raman mode in nanocrystalline diamond. Physical Review B, 2001. 63(12).

40. Hsu, Y.K., et al., Reversible phase transformation of $\mathrm{MnO} 2$ nanosheets in an electrochemical capacitor investigated by in situ Raman spectroscopy. Chemical Communications, 2011. 47(4): p. 1252-1254.

41. Pichler, T., et al., Localized and delocalized electronic states in single-wall carbon nanotubes. Physical Review Letters, 1998. 80(21): p. 4729-4732.

42. Queralto, A., et al., Laser-induced metal organic decomposition for Ce0.9Zr0.1O2-y epitaxial thin film growth. Journal of Alloys and Compounds, 2013. 574: p. 246-254.

43. Gyoergy, E., et al., Effect of laser radiation on multi-wall carbon nanotubes: study of shell structure and immobilization process. Journal of Nanoparticle Research, 2013. 15(8).

44. Singh, G., et al., Laser-induced exfoliation of amorphous carbon layer on an individual multiwall carbon nanotube. Applied Physics Letters, 2007. 91(3).

45. Kichambare, P.D., et al., Laser irradiation of carbon nanotubes. Materials Chemistry and Physics, 2001. 72(2): p. 218-222.

46. Salunkhe, R.R., et al., Rational design of coaxial structured carbon nanotubemanganese oxide (CNT-MnO2) for energy storage application. Nanotechnology, 2015. 26(20).

47. Tan, D.Z.W., et al., Controlled synthesis of MnO2/CNT nanocomposites for supercapacitor applications. Materials Technology, 2014. 29(A2): p. A107-A113. 